\title{
Cenozoic plate driving forces
}

\author{
C. Lithgow-Bertelloni \\ Institut für Geophysik, Universität Göttingen, Göttingen, Germany
}

Mark A. Richards

Dept. of Geology and Geophysics, University of California, Berkeley

\begin{abstract}
Past studies of plate driving forces have concluded that the forces due to subducted slabs in the upper mantle and those due to the thickening of the oceanic lithosphere are the principal driving forces. We reexamine the balance of driving forces for the present-day and extend our analysis through the Cenozoic, using an analytical torque balance method which accounts for interactions between plates via viscous coupling to the induced mantle flow. We use an evolving mantle density heterogeneity field based on the last $200 \mathrm{Myr}$. of subduction to drive plate motions, an approach which has proven successful in predicting the present-day mantle heterogeneity field. We find that for plausible upper mantle viscosities the forces due to subducted slabs in the Cenozoic and Mesozoic account for in excess of $90 \%$ of plate driving forces and those due to lithospheric thickening for less than $10 \%$.
\end{abstract}

\section{Introduction}

Understanding global plate motions is one of the central problems of geodynamics, and determining the balance of forces acting on plates remains one of the ultimate goals of mantle convection modeling. Previous studies have concluded that the plates are mainly driven by a combination of the "pull" of slabs on subducting plates and the "push" from the ridges, opposed by basal drag and collisional resistance at plate boundaries. What has been called the "ridge push" force is actually distributed over the entire area of oceanic plates and results from the thermal thickening of the dense oceanic lithosphere [Lister, 1975; Hager \& O'Connell, 1981]. "Slab pull" is also a buoyancy force due to the dense downgoing slab, which is coupled to the lithosphere by some combination of elastic and viscous forces in the slab and surrounding mantle.

Most previous studies have used a process of trial and error or inversion of plate velocities and the intraplate stress field to estimate the relative magnitude of driving and resisting torques [Solomon et al., 1975; Forsyth \& Uyeda, 1975; Chapple \& Tullis, 1977; Gordon et al., 1978; Richardson et al., 1979; Richardson, 1992]. In this work we use a forward modeling approach to predict plate motions. Our starting point is a physical model of plate driving forces, with plates viscously coupled to a continuously evolving model of density heterogeneity in the Earth's mantle and lithosphere. This approach, first used by Hager \& O'Connell [1981], has distinct advantages over empirical models: The density heterogeneity field can be compared with the structure of the mantle inferred from

Copyright 1995 by the American Geophysical Union.

Paper number 95GL01325

0094-8534/95/95GL-01325\$03.00 seismic tomography and, when convolved with the appropriate dynamical response functions, can be used to predict not only plate velocities [Ricard \& Vigny, 1989] but also the geoid [Ricard et al., 1993], dynamic topography and continental flooding [Gurnis, 1993]. Our model differs from other forward models based on density heterogeneity fields in two respects. First, we assume that density heterogeneity below the lithosphere is subducted material, so that our models are based on geologically constrained past subduction histories and lithospheric heterogeneity rather than being inferred from seismic tomography [e.g. Ricard \& Vigny, 1989; Woodward et al., 1991]. The second difference is that our approach allows us to predict past plate velocities.

We solve for the instantaneous 3-D flow and viscous stresses induced by the internal density contrasts arising from the subducted lithosphere, and those arising from a thickening oceanic lithosphere. Solutions are obtained using an analytical torque balance method similar to that of Hager \& O'Connell [1981]. We present calculations at the end of each of six stages of the Cenozoic and assess the success of our model by comparing predicted and observed plate velocities, along with a measure of estimated uncertainties. We examine the relative force balance between subducted slabs and lithospheric thickening for the entire Cenozoic. Finally, we investigate the effect of mantle viscosity structure on the relative magnitude of plate driving forces.

\section{Model and Methodology}

The forces that drive plate motions arise from buoyancy forces due to internal density heterogeneities. Near the surface, such forces include the thermal thickening of the oceanic lithosphere. In the deep mantle, it is likely that subducted slabs contribute the largest buoyancy forces [e.g. Richards \& Engebretson, 1992]. In our model these density heterogeneities act on the plates through the viscous mantle flow which they induce, and we ignore elastic forces. The resisting forces arise from viscous drag beneath the lithosphere, and we ignore local interplate forces such as collisional resistance at plate boundaries. Our goal is to construct the most straightforward possible model of plate motions which is consistent with a broad range of geological and geophysical constraints on global plate motions and mantle density structure.

Our model for the density heterogeneity field of the Earth's mantle is constructed from subduction histories for the 200 Myr. preceding the end of each plate stage of the Cenozoic reconstructions of Gordon \& Jurdy [1986]. We use their plate boundaries and velocities for the Cenozoic and our own compilations for the Mesozoic [Lithgow-Bertelloni et al., 1993]. Subducted slabs are assigned density contrasts according to an oceanic lithosphere cooling model, and these density con- 
trasts are prescribed to sink vertically through the upper mantle at the initial rate of convergence. We assume that the slabs freely penetrate through the $670 \mathrm{~km}$ discontinuity into the lower mantle, sinking at $1 / 4$ the upper mantle rate. This slowing factor is equal to the inverse of the natural logarithm of the viscosity contrast between upper and lower mantle [Gurnis \& Davies, 1986; Richards, 1991] for our preferred viscosity model (Table 1). With these assumptions, our model gives excellent agreement with the observed geoid and plate velocities [Ricard et al., 1993].

The effects of thickening of the oceanic lithosphere as a function of age are based on the reconstructed isochrons of Royer et al. [1993]. The density contrast in $\mathrm{kg} / \mathrm{m}^{3}$ is given by $(80 h-4) /(h+8)$, where the lithospheric plate thickness in $\mathrm{km}$ is $h=10 \tau^{1 / 2}$, and the age of the plate $\tau$ is in Myr. This corresponds to an $8 \mathrm{~km}$ thick oceanic crust of density $2900 \mathrm{~kg} / \mathrm{m}^{3}$ and a suboceanic lithospheric mantle of density $3380 \mathrm{~kg} / \mathrm{m}^{3}$.

We predict plate motions by computing the driving torques and then finding plate rotation poles such that the resisting torques exactly balance them. What allows us to predict the plate rotation vectors is the fact that the resisting torques are linearly dependent on them, while the driving torques are independent. The torque balance is expressed as $\mathbf{Q}_{i n t}=\mathbf{M} \vec{\omega}$, where $\vec{\omega}$ and $\overrightarrow{\mathbf{Q}}_{\text {int }}$ are $3 N$ dimensional vectors ( $N$ is the number of plates) containing, respectively, the 3-component Euler rotation vector of each plate and the driving vector torques acting on each plate. For each plate, $P$, we obtain $\overrightarrow{\mathbf{Q}}_{\text {int, }}$ from $\overrightarrow{\mathbf{Q}}_{\text {int, }}=\int_{A} \overrightarrow{\mathbf{r}} \times \vec{\tau}_{\text {int }} d A$, where $\vec{\tau}_{\text {int }}$ is the basal shear stress on each plate obtained with the propagator matrix solution of Hager \& $O^{\prime}$ Connell [1981], $\overrightarrow{\mathbf{r}}$ is the position vector and $A_{p}$ is the area of the plate. The $3 N \times 3 N$ matrix $\mathbf{M}$ relates the resisting torques to the Euler rotation vectors. It depends on the viscosity structure and contains information on the plate geometry. Its off-diagonal elements are non-zero because movement of any one plate will generate viscous stresses at the base of all the others through the induced mantle flow.

The problem thus consists of two parts whose solutions can be superposed, because they are both subject to the same type of boundary condition: 1) To calculate the driving shear stresses $\vec{\tau}_{\text {int }}$ induced by the density heterogeneity field the surface velocities are set to zero. 2) The resisting shear stresses are calculated by imposing the plate geometry and applying unit rotations in each of the three cartesian directions to every plate, in the absence of internal loads. An exact solution for plate motions (with zero net torque on each plate) is obtained by superposition of these two solutions. This is equivalent to the method of Ricard \& Vigny [1989].

When modeling piecewise continuous plate motions a technical problem arises due to the viscous stress singularity that forms at a "fluid" plate boundary [Hager \& O'Connell, 1981]. Viscous resistance at plate edges increases (unphysically) as the logarithm of the highest harmonic degree retained in the flow field. We truncate our calculations at degree 20 as Ricard \& Vigny [1989] and Ricard \& Wuming [1991]. We find that truncations at higher degrees (up to 50) do not change the results significantly, i.e. the correlation coefficients between predicted and observed plate motions change by less than 0.01 and the best fitting absolute viscosity by less than $15 \%$.

We have calculated solutions for the viscosity structures given in Table 1 . Our preferred viscosity structure (a) has a lithosphere which is 10 times more viscous than the upper mantle and a lower mantle which is 50 times more viscous. This viscosity structure gives the best fit to the observed geoid using our mantle density heterogeneity model [Ricard et
Table 1: Viscosity (Pa-s) ( $\eta$ ) Structure Models: (a) Fit to the geoid; (b) LVC $-300 \mathrm{~km}$ thick; (c) LVC $70 \mathrm{~km}$ thick; (d) Hager \& O'Connell [1981]

\begin{tabular}{rccccc}
\multicolumn{1}{c}{ Depth } & (a) & (b) & (c) & \multicolumn{1}{c}{ Depth } & (d) \\
\hline $0-130$ & $1 \times 10^{22}$ & $1 \times 10^{22}$ & $1 \times 10^{22}$ & $0-64$ & $1 \times 10^{21}$ \\
$130-200$ & $1 \times 10^{21}$ & $1 \times 10^{19}$ & $1 \times 10^{19}$ & $64-128$ & $1 \times 10^{19}$ \\
$200-410$ & $1 \times 10^{21}$ & $1 \times 10^{19}$ & $1 \times 10^{21}$ & $128-410$ & $1 \times 10^{21}$ \\
$410-670$ & $1 \times 10^{21}$ & $1 \times 10^{21}$ & $1 \times 10^{21}$ & $410-670$ & $1 \times 10^{21}$ \\
$670-2890$ & $5 \times 10^{22}$ & $5 \times 10^{22}$ & $5 \times 10^{22}$ & $670-2890$ & $1 \times 10^{21}$ \\
\hline
\end{tabular}

al., 1993]. The lithospheric viscosity is lower than that of the lower mantle, because it represents an average over relatively high viscosity regions beneath continents and old oceans, low viscosity regions beneath young oceans, and plate boundaries which appear to be very weak. Viscosity structures $b$ and $c$ are used to investigate the effects of a low viscosity channel.

The only free parameter in our model is the reference absolute viscosity, $\eta_{r}$. Plate velocities scale linearly with the absolute viscosity so that this parameter affects only plate speeds, not relative motion directions. The value of $\eta_{r}$ is determined by minimizing the $\chi^{2}$ misfit between the observed and predicted components of the linear momenta $v_{i j}=\iint\left(\vec{\omega}_{j} \times \vec{r}_{j}\right)_{i} d A / A_{j}$, where $i=x, y, z$, the index $j$ runs over the 12 plates, $r_{j}$ is the position vector, $\omega_{j}$ the rotation vector and $A_{j}$ the area of plate $j$.

\section{Analysis of Driving Forces}

Our model reproduces present day plate motions well, as shown by the comparison of the observed and predicted velocity fields in Figure 1. We quantify the agreement by computing the variance reduction of the horizontal divergence and radial vorticity fields as defined in Lithgow-Bertelloni et al. [1993] and global correlation coefficients between the predicted and observed cartesian components of the linear momenta.

We compute both weighted and unweighted linear correlation coefficients between observed and predicted velocity fields. For the former we weight the $V_{i j}$ 's by the fraction of the total surface area of the Earth that each plate occupies. This approach gives the global correlation between observed and predicted surface velocity fields, which we feel is the best measure of model success. For 36 degrees of freedom ( 3 cartesian components for each of 12 plates) the correlations are significant above the $95 \%$ confidence level for any correlation coefficient greater than 0.33 . The overall weighted correlation coefficient is $\sim .90$ (.62 unweighted). Our variance reduction for the horizontal divergence $(96 \%)$ and the radial vorticity $(66 \%)$ fields is substantially better than previous studies based on seismic tomography [Ricard \& Vigny, 1989; Woodward et al., 1993] which achieved instead $66 \%$ and $20 \%$ variance reduction, respectively. The good agreement suggests that flow induced by mass anomalies in the lithosphere and mantle accounts for most of the forces driving the plates, and that our density heterogeneity model maybe a good approximation to the structure of the mantle and lithosphere. The model is also successful at reproducing past plate motions with weighted correlation coefficients ranging from $0.7-0.9$ (.6-.8 unweighted) (Fig. 2). The decrease in the correlation coefficients for the stages postdating 43 Ma reflects an inability to reproduce the change in motion of the Pacific plate for these stages [Richards \& Lithgow-Bertelloni, 1995].

One deficiency of our model is that the velocities of plates with large continental areas are, in general, overpredicted. The magnitude and direction of the velocity of the Nazca and South 


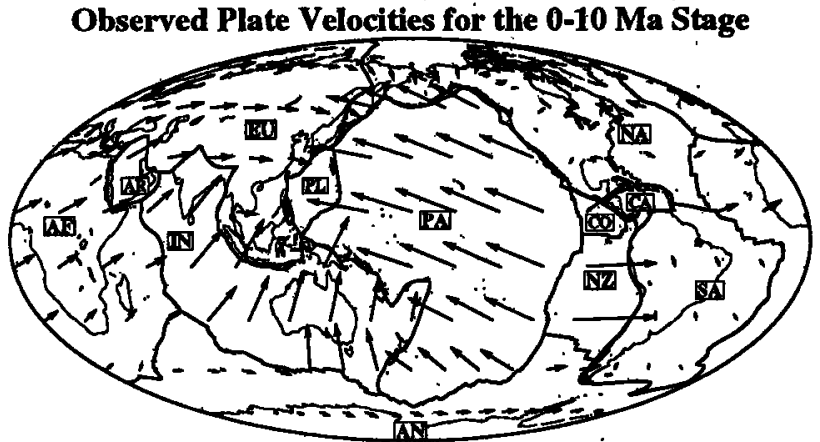

Predicted Plate Velocities for the 0-10 Ma Stage

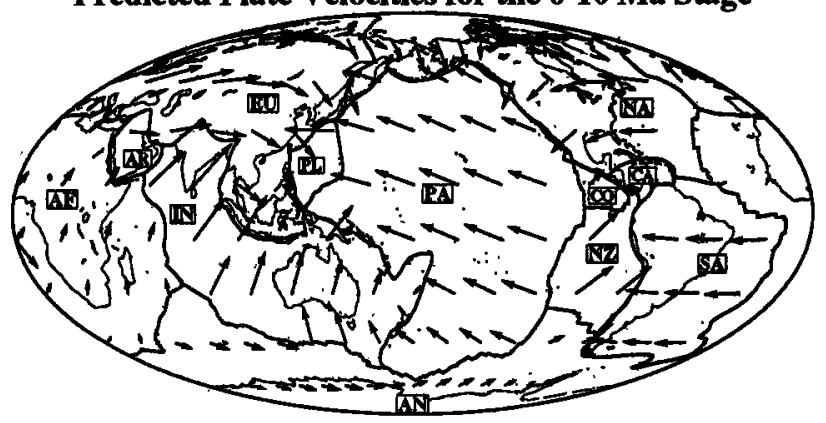

Figure 1. (Top) Present-day plate velocities and plate boundaries in the no-net rotation reference frame. The arrows are proportional to the magnitude of the plate velocity $(\longrightarrow=5 \mathrm{~cm} / \mathrm{yr})$. Plates: AF=Africa; AN=Antarctica AR=Arabia; $\mathrm{CA}=$ Caribbean; $\mathrm{CO}=\mathrm{Cocos} ; \mathrm{EU}=$ Eurasia; $\mathrm{IN}=$ India; $\mathrm{NA}=\mathrm{North}$ America; NZ=Nazca; PA=Pacific; $P L=$ Philippine; $S A=$ South America; (Bottom) Predicted plate velocities in the no-net torque reference frame.

American $\mathrm{p}^{1-r e s}$ are poorly predicted. The source of the discrepancies 1....y be due to: 1) approximate treatment of subduction dynamics; 2 ) other sources of density heterogeneities not included in the model; 3) unmodeled forces such as collisional resistance or the elastic component of slab pull.

We now analyze in detail the relative magnitudes of the plate driving forces (Fig. 3a). We separate the effects of each load: Lithospheric thickening, and the upper and lower mantle slabs are each allowed to drive the plates in the absence of the others. For each load we calculate the magnitude of the torques acting on each plate, using the same upper mantle viscosity structure, and calculate its percentage contribution.

The total contribution of upper mantle plus lower mantle slabs is in excess of $95 \%$ for all times, while that from lithospheric effects is of order $5 \%$ or less. The large value of our slab contribution is in large part due to the lower mantle slabs in our density heterogeneity model. The slight decrease in the contribution of the forces due to lithospheric thickening prior to the present stage is partially an artifact of the paucity of isochron data as we go back in time. The lack of data tends to lower the estimated age contrast, and therefore the thickness and density contrast, across an oceanic plate. If we include only the upper mantle slabs the total contribution from lithospheric thickening is $\sim 12-15 \%$ for our preferred viscosity structure. Using the viscosity structure of Hager \& $O^{\prime}$ Connell [1981] (Table 1) changes the relative contribution to $\sim 70 \%$ for the slabs and $-30 \%$ for lithospheric thickening, in agreement with their results. Including the lower mantle slabs yields a total contribution in excess of $95 \%$ for the slab component.

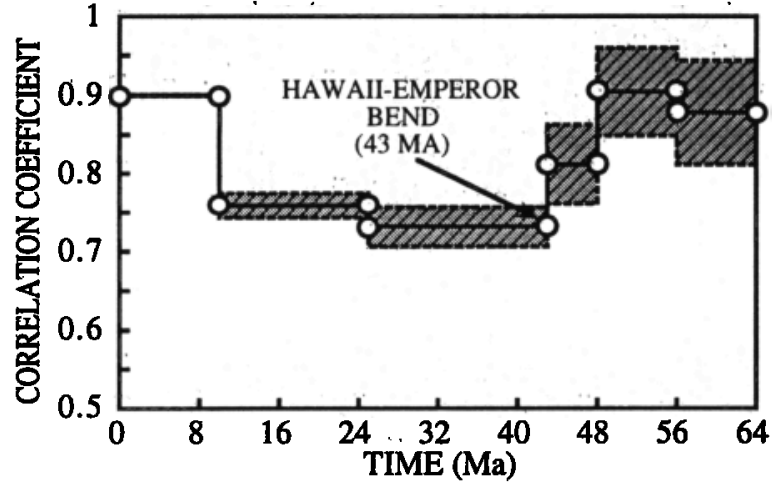

Figure 2. Weighted correlation coefficients between the observed and predicted components of plate velocities and associated $1 \sigma$ confidence contours (hatched areas). Circles mark the beginning and end of each stage. Predicted velocities, in the no-net torque reference frame, are compared to observed velocities in the no-net rotation reference frame, obtained by subtracting the net rotation of the lithosphere with respect to the mantle from the poles of rotation of Gordon \& Jurdy [1986].

(This viscosity structure produces very poor fits to the observed geoid.)

A contribution to plate forces might arise from the inherent density difference between continents and oceans.
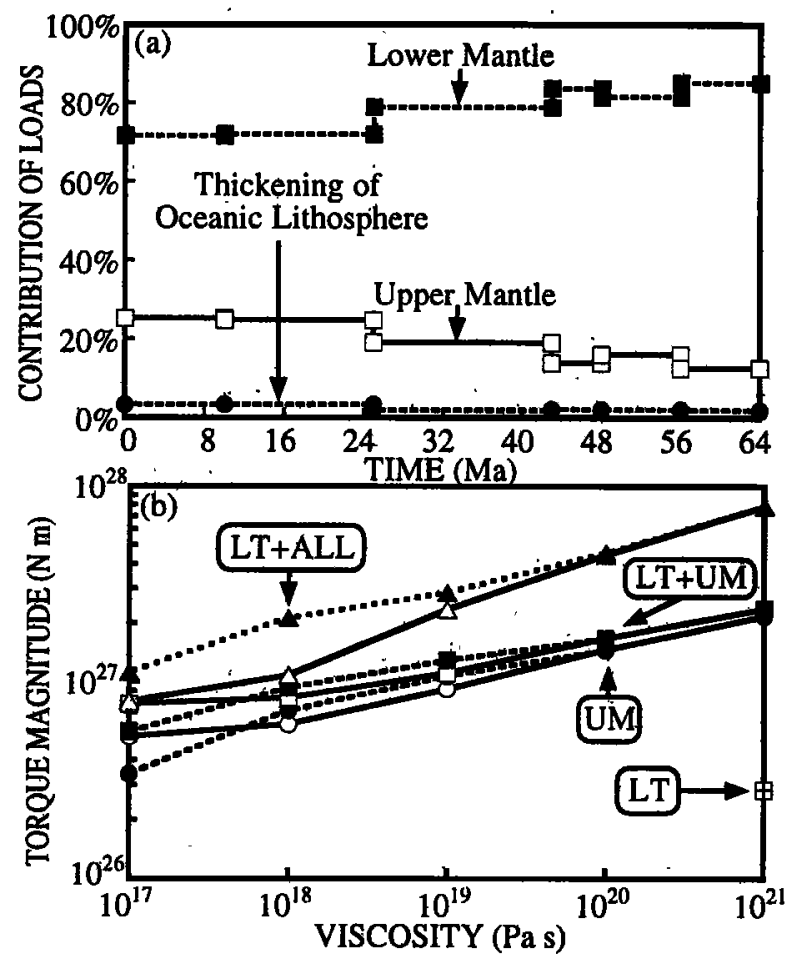

Figure 3(a) Percent contribution of driving loads as a function of time; (b) Effects of an increasingly less viscous low viscosity channel (LVC) on the magnitude of the torques exerted by: all the slabs (ALL) + lithospheric thickening (LT) (solid and open triangles), upper mantle slabs (UM) + LT (open and solid squares) and UM alone (open and solid circles). The magnitude of the torque exerted by LT alone is shown for reference. Solid lines and open symbols for viscosity structure (b) in Table 1; dashed lines and solid symbols for (c). 
Unfortunately, its effect is difficult to assess. If continents are rigid then there is no contribution to the driving torques. If, on the other hand, we consider the tendency towards gravitational spreading, then continents contribute a large negative mass anomaly, which will tend to oppose the effect of thickening of the oceanic lithosphere. [Franck, 1972; Hager \& $O ' C o n n e l l, 1981]$. In the latter case, the difference between oceanic and continental density structure contributes a somewhat larger torque on the plates than lithospheric thickening. Here we have, for simplicity, ignored the ocean-continent function.

To examine the sensitivity of our conclusions to the assumed viscosity structure, we investigate the effects of a low viscosity asthenospheric channel. This type of viscosity structure maximizes the relative contribution of lithospheric thickening to plate driving forces by partially decoupling the overlying lithosphere from the deep mantle flow induced by subduction-related density heterogeneity; in the inviscid limit, flow induced by mass anomalies lying below the lithosphere will exert no torque on the plates. Fig. $3 b$ shows the various plate driving torque contributions as functions of the viscosity of a low viscosity channel. To obtain a lithospheric thickening contribution to plate driving forces equal in magnitude to that of upper mantle slabs requires more than 3 orders of magnitude viscosity contrast between the upper mantle transition zone and the low viscosity channel. In other words, the viscosity of the low viscosity channel must be lower than $10^{17} \mathrm{~Pa}-\mathrm{s}$ and the upper mantle viscosity must be lower than $10^{20} \mathrm{~Pa}$-s. This result holds for a low viscosity channel either $\sim 100$ or $-300 \mathrm{~km}$ in thickness. Given the estimates from postglacial rebound on the viscosity of the upper mantle [Nakada \& Lambeck, 1987; Mitrovica \& Peltier, 1993] and a possibly "weak asthenosphere", these values are unreasonably low.

\section{Summary}

We have assumed that plate driving forces arise from the flow induced by density heterogeneities in the mantle due to slabs and horizontal differences in lithospheric density structure. We have constructed a simple model for mantle density heterogeneity based on the last $200 \mathrm{Myr}$. of subduction. With this model we predict present and past plate motions in excellent agreement with observations.

Examining the relative contribution of plate driving forces we have shown that the plate driving torques due to subducted slabs are in excess of $95 \%$, and lithospheric contributions only of order $5 \%$. To achieve a 1:1 ratio between lithospheric thickening forces and the forces due to subducted slabs, requires a low viscosity channel many orders of magnitude less viscous than the deep upper mantle. We conclude that plates are mostly driven by buoyancy forces due to subducted lithosphere and that these forces can be modeled with reasonable accuracy through the Cenozoic. Improved plate motion models require a better characterization of resisting forces along plate boundary faults.

Acknowledgments. We thank R. Richardson and two anonymous referees for thoughtful reviews, N. Ribe and U. Christensen for helpful discussions. This work was partly supported by NSF grant EAR-9117538 and $\mathrm{CH} 77-4-3$ to U. C. from the Deutsche Forschungs Gemeinschaft.

\section{References}

Chapple, W. M. and T. E. Tullis, Evaluation of the forces that drive the plates, J. Geophys. Res., 82, 1967-1984, 1977.
Forsyth, D. W. and S. Uyeda, On the relative importance of driving forces of plate motions, Geophys. J. R. Astro. Soc., 43, 163-200, 1975.

Franck, F. C., Plate tectonics, the analogy with glacial flow, and isostasy, in Flow and Fracture of Rocks, Geophysical Monograph Series, 16, edited by H. C. Heard, I. Y. Borg, N. L. Carter, and C. B. Raleigh, 285-292, AGU, Washington, DC, 1972.

Gordon, R. G., A. Cox, and C. E. Harter, Absolute motion of an individual plate estimated from its ridge and trench boundaries, Nature, 274, 752-755, 1978.

Gordon, R. G. and D. M. Jurdy, Cenozoic global plate motions, $J$. Geophys. Res., 91, 12,389-12,406, 1986.

Gurnis, M. and G. F. Davies, Numerical models of high Rayleigh number convection in a medium with depth-dependent viscosity, Geophys. J. R. Astro. Soc., 85, 523-541, 1986.

Gurnis, M., Phanerozoic marine inundation of continents driven by dynamic topography above subducting slabs, Nature, 364, 589-593, 1993.

Hager, B. H. and R. J. O'Connell, A simple global model of plate dynamics and mantle convection, J. Geophys. Res., 86, 4843-4867, 1981.

Lister, C. R. B., Gravitational drive on oceanic plates caused by thermal contraction, Nature, 257, 663-665, 1975.

Lithgow-Bertelloni, C., M. A. Richards, Y. Ricard, R. J. O'Connell, and D. C. Engebretson, Toroidal-poloidal partitioning of plate motion since $120 \mathrm{Ma}$, Geophys. Res. Lett., 20, 357-378, 1993.

Mitrovica, J. X. and W. R. Peltier, The inference of mantle viscosity from an inversion of the Fennoscandian relaxation spectrum, Geophys. J., 114, 45-62, 1993.

Nakada, M. and K. Lambeck, Glacial Rebound and relative sea-level variations: a new appraisal, Geophys. J., 90, 171-224, 1987.

Ricard, Y., M. Richards, C. Lithgow-Bertelloni, Y. Le Stunff, A geodynamical model of mantle density heterogeneity, J. Geophys. Res., 98, 21895-21909, 1993.

Ricard, Y. and C. Vigny, Mantle dynamics with induced plate tectonics, J. Geophys. Res., 94, 17,543-17,560, 1989.

Ricard, Y. and B. Wuming, Inferring the viscosity and the 3-D density structure of the mantle from geoid, topography and plate velocities, Geophys. J. Int., 105, 561-571, 1991.

Richards, M. A., Hotspots and the case for a high-viscosity lower mantle, in Glacial Isostasy, Sea Level and Mantle Rheology, eds. R. Sabadini et al., 571-588, Kluwer Academic, Hingham, Mass., 1991.

Richards, M. A. and D. C. Engebretson, Large-scale mantle convection and the history of subduction, Nature, 355, 437-440, 1992.

Richards, M. A. and C. Lithgow-Bertelloni, Plate motion changes, the Hawaiian-Emperor bend, and the apparent success of dynamical models, Science, (submitted).

Richardson, R. M., S. C. Solomon, and N. H. Sleep, Tectonic stress in the plates, Rev. Geophys. Space Phys., 17, 981-1019, 1979.

Richardson, R. M., Ridge Forces, absolute plate motions, and the intraplate stress field, J. Geophys. Res., 97, 11739-11748, 1992.

Royer, J.-Y., R. D. Müller, L. M. Gahagan, L. A. Lawver, C. L. Mayes, D. Nürnberg, and J. G. Sclater, A global isochron chart, University of Texas Institute for Geophys. Technical Report No. 117, pp. 38, 1992.

Solomon, S. C., N. H. Sleep, and R. M. Richardson, On the forces driving plate tectonics: Inference from absolute plate velocities and intraplate stress, Geophys. J. R. Astro. Soc., 42, 769-801, 1975.

Woodward, R. L., A. M. Forte, W. J. Su, and A. M. Dziewonski, Constraints on the large-scale structure of the Earth's mantle, Evolution of the Earth and Planets, Geophys. Monograph 74, eds. E. Takahashi, R. Jeanloz and D. Rubie, Washington, pp. 89-109, 1993.

Carolina Lithgow-Bertelloni, Institut für Geophysik, U. Göttingen, Herzberger Landstrasse 180, 37075 Göttingen, Germany.

Mark A. Richards, Dept. of Geology and Geophysics, 301 McCone Hall, University of California, Berkeley, CA 94720-4767.
(Received July 12, 1994; revised November 21, 1994; accepted February 5, 1995) 